\title{
Increased expression of long noncoding RNA TUG1 predicts a poor prognosis of gastric cancer and regulates cell proliferation by epigenetically silencing of p57
}

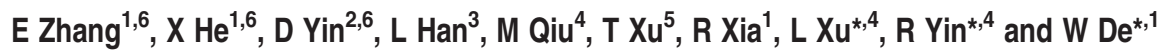

Recent evidence highlights long noncoding RNAs (IncRNAs) as crucial regulators of cancer biology that contribute to tumorigenesis. LncRNA TUG1 was initially detected in a genomic screen for genes upregulated in response to taurine treatment in developing mouse retinal cells. Our previous study showed that TUG1 could affect cell proliferation through epigenetically regulating HOXB7 in human non-small cell lung cancer. However, the clinical significance and potential role of TUG1 in GC remains unclear. In this study, we found that TUG1 is significantly increased and is correlated with outcomes in gastric cancer (GC). Further experiments revealed that knockdown of TUG1 repressed GC proliferation both in vitro and in vivo. Mechanistic investigations showed that TUG1 has a key role in G0/G1 arrest. We further demonstrated that TUG1 was associated with PRC2 and that this association was required for epigenetic repression of cyclin-dependent protein kinase inhibitors, including p15, p16, p21, p27 and p57, thus contributing to the regulation of GC cell cycle and proliferation. Together, our results suggest that TUG1, as a regulator of proliferation, may serve as a candidate prognostic biomarker and target for new therapies in human GC.

Cell Death and Disease (2016) 7, e2109; doi:10.1038/cddis.2015.356; published online 25 February 2016

Gastric cancer (GC) is one of the most common malignancies worldwide. ${ }^{1}$ Despite efforts to improve diagnostic techniques and patient management, there has been little progress toward improving the overall survival of GC patients. ${ }^{2}$ Gastric carcinogenesis is a complicated biological process, which results from the dysregulation of many tumor-related genes. Therefore, the identification of new biomarkers for GC and a better understanding of the molecular mechanisms underlying gastric carcinogenesis will improve the diagnosis and treatment of GC.

With the development of next-generation sequencing technologies, it was determined that long noncoding RNAs (IncRNAs) are pervasively transcribed in the genome., LncRNAs are a class of transcripts longer than 200 nucleotides with limited protein-coding potential. ${ }^{5}$ Recently, many studies have shown that IncRNAs could have critical roles in many biological processes including cellular development and differentiation. ${ }^{6-10}$ The aberrant expression of IncRNAs has also been shown in various types of disease, including cancer. ${ }^{11-16}$ For example, HOTAIR may be involved in the transcriptional repression of the HOX loci and promote breast metastasis by binding to polycomb repressive complex 2 (PRC2) ${ }^{13}$ In addition to regulation at the transcriptional level, IncRNAs can also serve as a 'sponge' to titrate microRNAs, thus participating in post-transcriptional processing. ${ }^{11,17}$ Our previous study showed that HOTAIR could also function as a competing endogenous RNA by sponging miR-331-3p in $\mathrm{GC}^{18}$

Recently, numerous IncRNAs have been identified to have a direct role in recruiting PRC2. PRC2, a methyltransferase that is composed of enhancer of zeste homolog 2 (EZH2), suppressor of zeste 12 (SUZ12) and embryonic ectoderm development, can catalyze the di- and trimethylation of lysine residue 27 of histone 3 (H3K27me3), thus modulating gene expression. These IncRNAs epigenetically regulate gene expression through binding to PRC2 in various biological processes, especially in cancer, such us HOTAIR and ANRIL. ${ }^{13,16}$

Khalil et al. ${ }^{19}$, by way of genome-wide RNA immunoprecipitation (RIP) analysis, identified that approximately $20 \%$ of the IncRNAs expressed in various cell types are bound to

\footnotetext{
${ }^{1}$ Department of Biochemistry and Molecular Biology, Nanjing Medical University, Nanjing, Jiangsu, China; ${ }^{2}$ Central Laboratory, The Second Affiliated Hospital of Southeast University, Nanjing, Jiangsu, China; ${ }^{3}$ Department of Oncology, Xuzhou Central Hospital, Affiliated Xuzhou Hospital, College of Medicine, Southeast University, Xuzhou, Jiangsu, China; ${ }^{4}$ Department of Thoracic Surgery, Jiangsu Key Laboratory of Molecular and Translational Cancer Research, Nanjing Medical University Affiliated Cancer Hospital, Cancer Institute of Jiangsu Province, Nanjing, Jiangsu, China and ${ }^{5}$ Department of Oncology, First Affiliated Hospital of Nanjing Medical University, Nanjing, Jiangsu, China

${ }^{*}$ Corresponding author: L Xu or R Yin, Department of Thoracic Surgery, Jiangsu Key Laboratory of Molecular and Translational Cancer Research, Nanjing Medical University Affiliated Cancer Hospital, Cancer Institute of Jiangsu Province, Baiziting 42, Nanjing, Jiangsu 210009, China. Tel: +86 02583284700 ; Fax: +86 025 83641062; E-mail: xulin_njmu@163.com or yinrong_njmu@163.com

or W De, Department of Biochemistry and Molecular Biology, Nanjing Medical University, Nanjing, Jiangsu 210009, China. Tel: +86 02586862728 ; Fax: +86 02586862728 ; E-mail: dewei_njmu@sina.com

${ }^{6}$ These authors contributed equally to this work.

Abbreviations: IncRNA, long noncoding RNA; TUG1, taurine upregulated gene 1; PRC2, polycomb repressive complex 2; GC, gastric cancer; EZH2, enhancer of zeste homolog 2; CKIs, cyclin-dependent protein kinase inhibitors; ChIP, chromatin immunoprecipitation; RIP, RNA immunoprecipitation

Received 28.6.15; revised 28.10.15; accepted 10.11.15; Edited by R Mantovani
} 
PRC2, including taurine upregulated gene 1 (TUG1). TUG1 was initially detected in a genomic screen for genes upregulated in response to taurine treatment in developing mouse retinal cells. The depletion of TUG1 in the developing mouse eye was found to block retinal development. ${ }^{20}$ In addition, dysregulation of TUG1 could participate in the progression of a variety of tumors. ${ }^{21-23}$ Our previous study also found that TUG1 could affect cell proliferation through epigenetically regulating $\mathrm{HOXB} 7$ by binding to $\mathrm{PRC} 2$ in human non-small cell lung cancer. ${ }^{24}$ However, the biological functions of TUG1 in the control of GC tumorigenesis have not been well characterized, which prompted us to explore the role of TUG1 in human GC.

In this study, we found that IncRNA TUG1 was significantly upregulated in GC tissues compared with the corresponding non-tumor lung tissues and may serve as an independent predictor for overall survival in GC. In addition, TUG1 knockdown repressed GC proliferation both in vitro and in vivo. Further experiments demonstrated that TUG1 was associated with PRC2 and that this association was required for the epigenetic repression of cyclin-dependent protein kinase inhibitors (CKIs), including p15, p16, p21, p27 and p57, thus contributing to the regulation of both the GC cell cycle and proliferation, which may partly account for TUG1-mediated proliferation regulation, thus affecting the proliferation of GC.

\section{Results}

TUG1 is upregulated in human GC tissues and is positively correlated with deeper tumor invasion depth and advanced TNM stage. The level of TUG1 was detected in 100 paired GC tissues and adjacent normal tissues using qRT-PCR with normalization to $\beta$-actin. As shown in Figure 1a, TUG1 expression was significantly upregulated in $85 \%$ (85 of 100) of cancerous tissues compared with normal controls $(6.0488 \pm 6.14159, P<0.01)$. Next, we used a $t$-test to examine the correlation of TUG1 expression level with the clinicopathological features in patients with GC. There was an obvious positive correlation between increased TUG1 levels and deeper tumor invasion depth $(6.6585 \pm 6.36480$ versus $2.5940 \pm 2.93578, \quad P=0.017)$ and advanced TNM stage $(8.3053 \pm 7.91956$ versus $4.3465 \pm 3.57530, P=0.001$ ) (Figures $1 \mathrm{~b}$ and $\mathrm{c}$ ). Furthermore, we divided the samples into high (above the median, $n=50$ ) and low (below the median, $n=50$ ) TUG1 expression groups according to the median value of TUG1 levels. A chisquare test was then performed to evaluate the clinicopathological features between the two groups. As shown in Table 1, the TUG1 level was also correlated with tumor invasion depth $(P=0.002)$ and TNM stage $(P=0.009)$. No relationship between TUG1 expression and other factors, for example, sex (male, female), age $(\leq 60,>60)$, histological grade (low or undiffer, moderate or high), lymph node metastasis (N0, N1 or above) or distant metastasis (M0, M1), was found in our study.

Overexpression of TUG1 predicts a poor prognosis and could be regarded as an independent predictor for overall survival of GC. To further evaluate the value of TUG1 in the prognosis of patients with GC, we used a
Kaplan-Meier survival analysis and log-rank tests. The median survival time was 54 months in the low TUG1 group, whereas it was 31 months in the high TUG1 group. Overexpression of TUG1 predicted a poor prognosis in patients with GC $(P=0.013)$. Univariate analysis identified four prognostic factors: lymph node metastasis (N0, N1 or above), TNM stage (I/II, III/IV), distant metastasis (M0, M1) and TUG1 expression. Multivariate analysis further revealed that TUG1 expression could be regarded as an independent predictor for overall survival in patients with $\mathrm{GC}(P=0.003)$, as well as TNM stage $(P=0.019)$ and lymph node metastasis $(P=0.001$; Table 2).

TUG1 regulates GC cell proliferation by affecting the cell cycle. To explore the role of high expression of TUG1 in GC, as shown in Figure 2a, we utilized four GC cell lines expressing higher levels of TUG1 than the normal gastric epithelial cell line (GES-1). Then, TUG1 siRNA was transfected into AGS and BGC-823 cell lines. To avoid offtarget effects, we used two effective interference target sequences of TUG1, as previously described. ${ }^{24}$ Q-PCR assays revealed that TUG1 expression was significantly reduced (Figure 2b). The MTT assays showed that knockdown of TUG1 expression significantly inhibited cell proliferation compared with the control cells (Figure 2c). Similarly, the result of colony formation assays revealed that clonogenic survival was significantly decreased following inhibition of TUG1 both in AGS and BGC-823 cell lines (Figure 2d). Next, flow cytometric analysis was performed to further examine the effect of TUG1 on the proliferation of GC cells by altering cell cycle progression. The results revealed that the cell cycle progression of si-TUG1 cells was significantly stalled at the G1-G0 phase compared with cells transfected with si-NC, both in AGS and BGC-823 cell lines (Figure 2e).

The impact of TUG1 on tumorigenesis in vivo. To further determine whether TUG1 affects tumorigenesis in vivo, shCtrl/shTUG1-transfected AGS cells were inoculated into nude mice. Consistent with the in vitro results, tumor growth in the shTUG1 group was obviously slower than that in the Scramble group (Figures $3 a$ and $b$ ). Up to 16 days after injection, the average tumor weight in the shTUG1 group was significantly lower than that in the control group (Figure 3c). qRT-PCR analysis was performed to detect the average expression of TUG1 in tumor tissues. The results showed that the average level of TUG1 in the shTUG1 group was lower than that in the control group (Figure 3c). Moreover, we also found that the tumors developed from control cells showed stronger Ki-67 expression than tumors formed from shTUG1 and that tumors that developed from shTUG1 cells showed a stronger p57 expression than tumors formed in the control, as detected by IHC analysis (Figure $3 \mathrm{~d}$ ). These data further supported the role of TUG1 in GC cell proliferation.

TUG1 was required for the epigenetic repression of CKIs by binding to PRC2, thus contributing to the regulation of the $\mathrm{GC}$ cell cycle and cell proliferation. To explore the fact that TUG1 has a role in G0/G1 arrest, we investigated the expression of CKIs, and the results showed that p15, p16, 

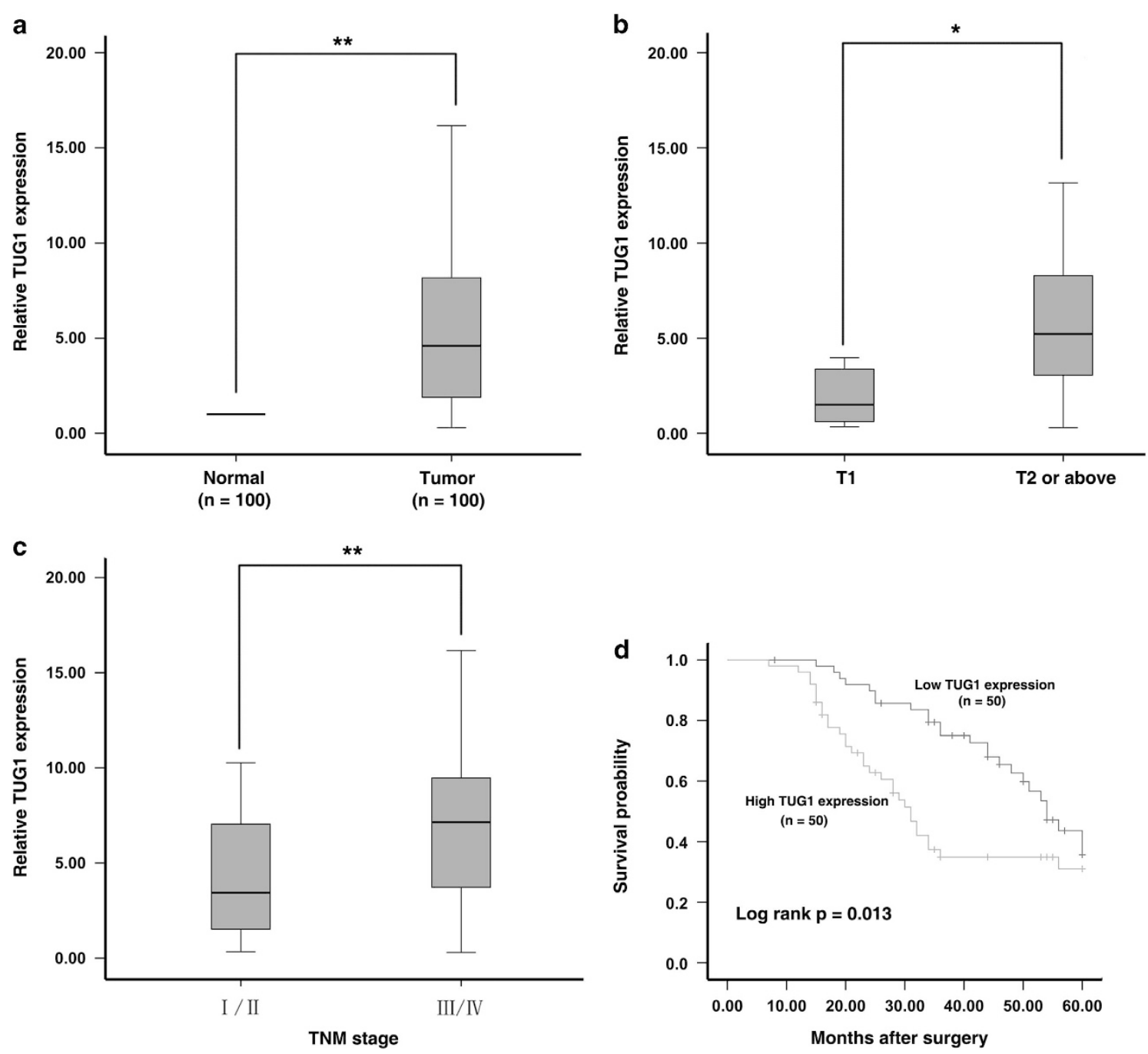

Figure 1 Expression of TUG1 in GC tissues and its clinical parameters. (a) Relative expression of TUG1 in GC tissues ( $N=100)$ compared with the corresponding nontumor tissues $(N=100)$. TUG1 expression was examined using quantitative real-time PCR (qRT-PCR) and normalized to $\beta$-actin expression. The results are presented as the fold-change in tumor tissues relative to normal tissues. (b and c) Higher TUG1 was positively correlated with advanced invasion depth and TNM stage. (d) Patients with high levels of TUG1 expression showed reduced survival times compared with patients with low levels of TUG1 expression. ${ }^{*} P<0.05$, ${ }^{* *} P<0.01$

p21, p27 and p57 were all obviously increased with knockdown of TUG1 (Figure 4a). To further study the mechanism of TUG1 in the regulation of the GC cell cycle, we measured TUG1 expression in nuclear and cytosolic fractions by qRTPCR. We found a considerable increase in TUG1 expression in the nucleus versus the cytosol (Figure 4b), suggesting that TUG1 may have a major regulatory function at the transcriptional level.

To further study the TUG1-associated regulation of GC cell proliferation, we tested whether TUG1 can bind PRC2 in GC cells. As shown in Figure 4c, the endogenous TUG1 was enriched in the anti-EZH2 RIP fraction relative to the input compared with the IgG fraction both in AGS and BGC-823 cell lines. Moreover, using an antibody specific to SUZ12, another member of the PRC2 complex, we also observed that endogenous TUG1 was obviously enriched in the anti-SUZ12 RNA-IP fraction (Figure 4c).
Next, the role of PRC2 in coregulating the suppression of TUG1-suppressed CKIs was investigated by EZH2 knockdown, and both were induced in cells transfected with si-EZH2 (Figure 5a). Similar results were observed for the knockdown of SUZ12 (Figure 5a). To avoid off-target effects, we used an interference target sequence against EZH2 and SUZ12, as studied in a previous article (Supplementary Figure S1A). ${ }^{25,26}$

To address whether TUG1 is involved in transcriptional repression through the enrichment of $\mathrm{EZH} 2$ to target gene promoters, we conducted chromatin immunoprecipitation (ChIP) analysis by TUG1 knockdown. The ChIP assays demonstrated that knockdown of TUG1 decreased the binding of EZH2 and H3K27me3 levels across the p15, p16, p21, p27 and p57 promoters (Figure 5b). As positive controls, no significant change was detected at the promoter of HOXA9, a gene regulated through $\mathrm{EZH} 2{ }^{27}$ The levels of $\mathrm{EZH} 2$ and SUZ12 were not affected by TUG1-knockdown cells. These 
results indicated that the decreases in PRC2 chromatin binding and H3K27me3 are mediated by TUG1-knockdown. These results suggest that TUG1 is required to target EZH2 occupancy and works to epigenetically modulate the expression of p15, p16, p21, p27 and p57.

The roles of EZH2 and p57 in GC. To verify the function of $\mathrm{EZH} 2$ in GC, immunohistochemistry was used to detect the expression of the EZH2 protein in 30 pairs of $\mathrm{GC}$ with the corresponding non-tumor tissues. All of the tumors showed positive immunostaining for EZH2 protein: 6 of the $30 \mathrm{GC}$ cases $(20 \%)$ showed weakly positive staining, and 24 GC cases $(80 \%)$ showed strongly positive staining. In contrast, all of the corresponding non-tumor tissues showed weakly positive immunostaining of EZH2 protein. The representative results are shown in Figure 6a. $\mathrm{EZH} 2$ was obviously upregulated in GC tissues. Further analysis showed that the expression of TUG1 was positively correlated with

Table 1 The clinicopathological factors of GC patients

\begin{tabular}{|c|c|c|c|}
\hline \multirow[t]{2}{*}{ Characteristics } & \multicolumn{2}{|c|}{ Expression of TUG1 } & \multirow[t]{2}{*}{$P$-value ${ }^{a}$} \\
\hline & Low & High & \\
\hline Sex & & & 0.84 \\
\hline Male & 29 & 28 & \\
\hline Female & 21 & 22 & \\
\hline Age & & & 0.841 \\
\hline$\leq 60$ & 25 & 24 & \\
\hline$>60$ & 25 & 26 & \\
\hline Histological grade & & & 0.161 \\
\hline Low or undiffer & 23 & 30 & \\
\hline Middle or high & 27 & 20 & \\
\hline Tumor invasion depth $(T)$ & & & $0.002^{* *}$ \\
\hline T1 & 13 & 2 & \\
\hline T2 or above & 37 & 48 & \\
\hline Lymph node metastasis $(\mathrm{N})$ & & & 0.545 \\
\hline No & 23 & 20 & \\
\hline N1 or above & 27 & 30 & \\
\hline Distant metastasis (M) & & & 0.646 \\
\hline MO & 48 & 47 & \\
\hline M1 & 2 & 3 & \\
\hline TNM stage & & & $0.009^{\star *}$ \\
\hline $\mathrm{I} / \mathrm{II}$ & 35 & 22 & \\
\hline III/IV & 15 & 28 & \\
\hline
\end{tabular}

${ }^{\text {a Chi-square test. }{ }^{* *} P<0.01}$
EZH2 protein levels in GC tissues (Figure 6a). Moreover, flow cytometric analysis demonstrated that the cell cycle progression of si-EZH2 cells was stalled at the G1 phase compared with cells transfected with si-NC (Figure 6b).

The functional roles of p15, p16 and p21 have been previously illustrated in GC. ${ }^{28,29}$ Our previous research suggested that p27 serves as a tumor suppressor in GC. ${ }^{30}$ However, the functional role of p57 in GC remains unclear. First, qRT-PCR was used to detect the expression of p57 in 30 pairs of GC and corresponding non-tumor tissues. As shown in Figures 6c, p57 was obviously downregulated in GC tissues. In addition, the results of western blot assays showed that p57 was obviously increased with the knockdown of TUG1 and EZH2 (Figure 6c). Overexpression of p57 could induce growth inhibition and G1-G0 phase arrest (Figure 6c). Moreover, to further prove the relationships between TUG1 and p57, as shown in Figure 6c, co-transfection of p57 and si-TUG1 promoted TUG1-knockdown-mediated G1-G0 phase arrest. In addition, knockdown of p57 could partly reverse TUG1-knockdown-mediated growth inhibition. TUG1/ p21 double knockdown could exert the same effect. The siRNAs could effectively downregulate the expression of p57 and p21 (Supplementary Figure S1B).

\section{Discussion}

To date, the newly discovered IncRNAs have emerged as important factors in cellular development and human diseases. In this study, we found that the average level of TUG1 in GC tissues was significantly higher than in corresponding nontumor tissues. The high expression level of TUG1 in GC patients was positively correlated with invasion depth and TNM stage. Moreover, high TUG1 expression in GC tissues was associated with a poor prognosis and could be an independent prognostic indicator. These results suggested that TUG1 may have an important role in GC progression. Previous studies found that TUG1 was upregulated in urothelial carcinoma of the bladder, osteosarcoma and esophageal squamous cell carcinoma. ${ }^{21-23}$ However, our previous study found that TUG1 is downregulated in NSCLC. ${ }^{24}$ This finding is probably because IncRNAs exhibit remarkably tissue-specific expression patterns compared with protein-coding genes and indicates that TUG1 may have a tissue-specific expression

Table 2 Univariate and multivariate analyses of the clinicopathological factors for overall survival in 100 patients with GC

\begin{tabular}{|c|c|c|c|c|c|c|}
\hline \multirow[t]{2}{*}{ Risk factors } & \multicolumn{3}{|c|}{ Univariate analysis } & \multicolumn{3}{|c|}{ Multivariate analysis } \\
\hline & HR & $P$-value & $95 \% \mathrm{Cl}$ & HR & $P$-value & $95 \% \mathrm{Cl}$ \\
\hline TUG1 expression & 1.091 & $<0.001^{\star *}$ & $1.048-1.137$ & 1.066 & $0.003^{\star \star}$ & $1.023-1.112$ \\
\hline Lymph node metastasis (N0, N1 or above) & 2.912 & $<0.001^{\star *}$ & $1.609-5.270$ & 2.697 & $0.001^{\star *}$ & $1.471-4.946$ \\
\hline TNM stage (I/II, III/IV) & 2.685 & $<0.001^{\star *}$ & $1.573-4.583$ & 2.005 & $0.019^{*}$ & $1.123-3.579$ \\
\hline Distant metastasis (M0, M1) & 4.167 & $0.007^{\star *}$ & $1.469-11.820$ & 2.52 & 0.092 & $0.859-7.393$ \\
\hline Histological grade (low or undiffer, middle or high) & 0.676 & 0.15 & $0.397-1.152$ & & & \\
\hline Age $(\leq 60,>60)$ & 1.182 & 0.533 & $0.698-2.001$ & & & \\
\hline Tumor invasion depth (T1, T2 or above) & 1.863 & 0.152 & $0.796-4.360$ & & & \\
\hline Sex (male, female) & 1.473 & 0.166 & $0.852-2.546$ & & & \\
\hline
\end{tabular}

${ }^{\star} P<0.05 .{ }^{* *} P<0.01$

Abbreviation: $\mathrm{HR}$, hazard ratio 

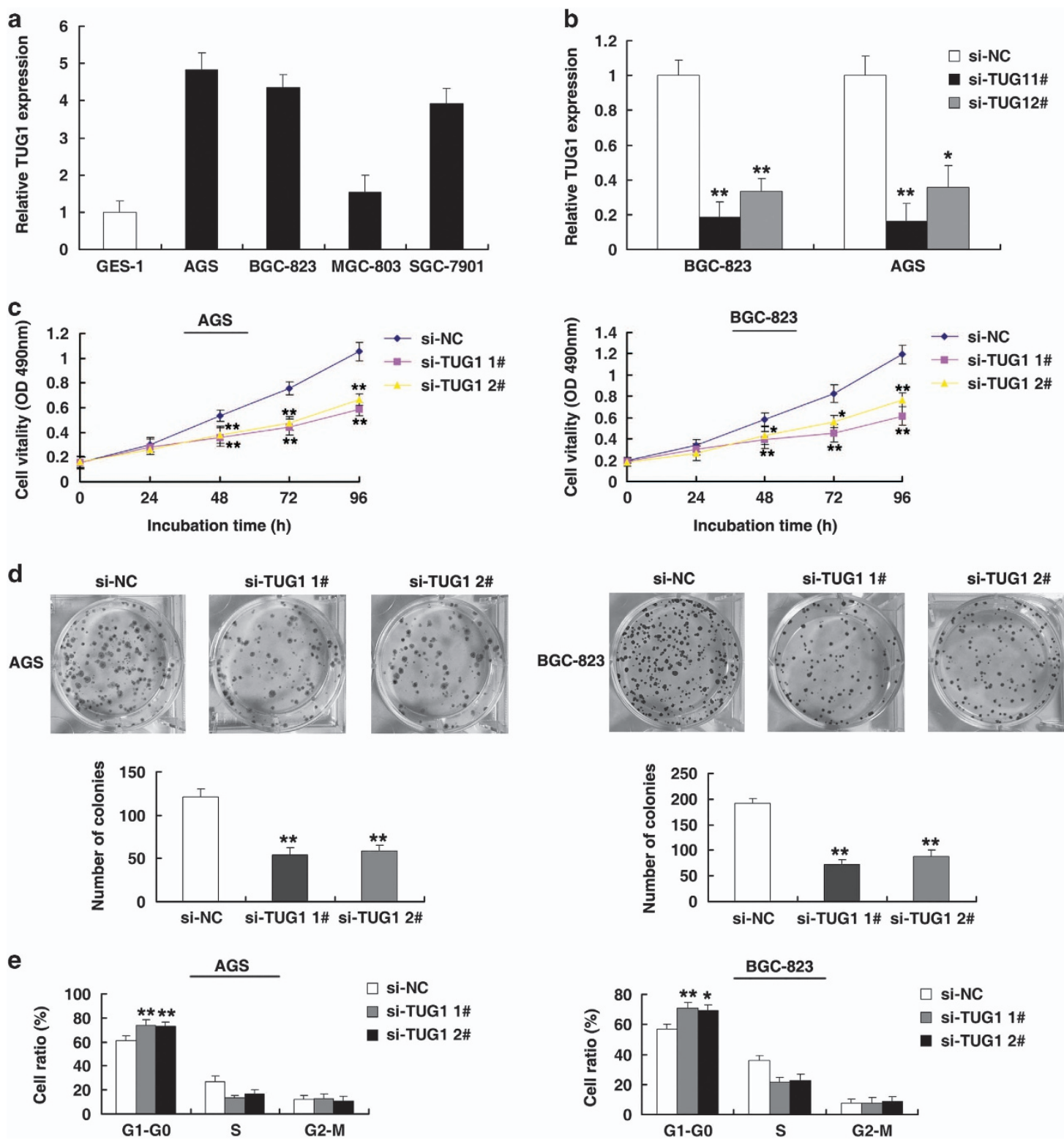

Figure 2 TUG1 regulates GC cell proliferation in vitro. (a) Analysis of TUG1 expression levels in GC cell lines (AGS, BGC-823, MGC-803 and SGC-7901) compared with a normal gastric epithelium cell line (GES-1) by qRT-PCR. (b) The relative expression level of TUG1 in GC cells, transfected with si-NC or si-TUG1 (si-TUG1\#1 and \#2), was tested using GPCR. (c) MTT assays were performed to determine cell proliferation of AGS and BGC-823 cells after transfection of siRNA against TUG1. (d) The representative results of colony formation of AGS and BGC-823 cells transfected with siRNA against TUG1. (e) At $48 \mathrm{~h}$ after transfection, the cell cycle was analyzed by flow cytometry. The bar chart represents the percentage of cells in G1-G0, S, or G2-M phase, as indicated. ${ }^{*} P<0.05,{ }^{* *} P<0.01$

pattern. ${ }^{31,32}$ Moreover, Cao et al. ${ }^{33}$ found that TUG1 was upregulated in GC by analysis of IncRNA expression profiles from Gene Expression Omnibus (GEO) in GC. Our results validated the expression results of TUG1 using microarrays with GC tissues and suggested an important role of TUG1 in GC development and progression. In addition, aberrant expression of IncRNAs may be involved in the progression of multiple tumors and can be used as a prognostic indicator. ${ }^{13,34,35}$ Our previous studies also showed that the IncRNAs ANRIL, HOTAIR and TINCR could serve as prognostic factors in GC. . $^{16,18,36}$
Although TUG1 has been studied in a variety of physiological and pathological processes, the possible role of TUG1 in GC remains to be clarified. In our study, the function of TUG1 was investigated by RNA interference (RNAi)-mediated knockdown. As a result, inhibition of TUG1 could promote NSCLC cell proliferation both in vitro and in vivo. Moreover, the knockdown of TUG1 could induce obvious G0/G1 arrest.

Khalil et al. ${ }^{19}$ found that TUG1 could have an important role in the cell cycle of normal cells by binding to PRC2. Our prior study showed that TUG1 also regulated the cell cycle during lung cancer. ${ }^{24}$ In human cancers, overactivation of 
a

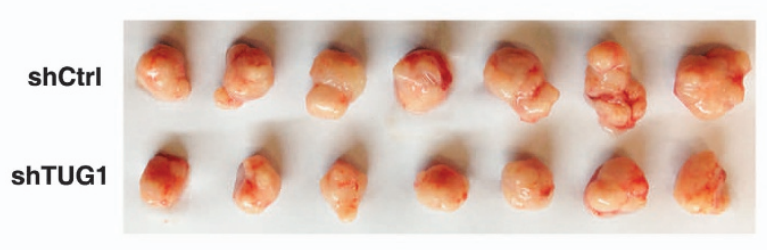

b
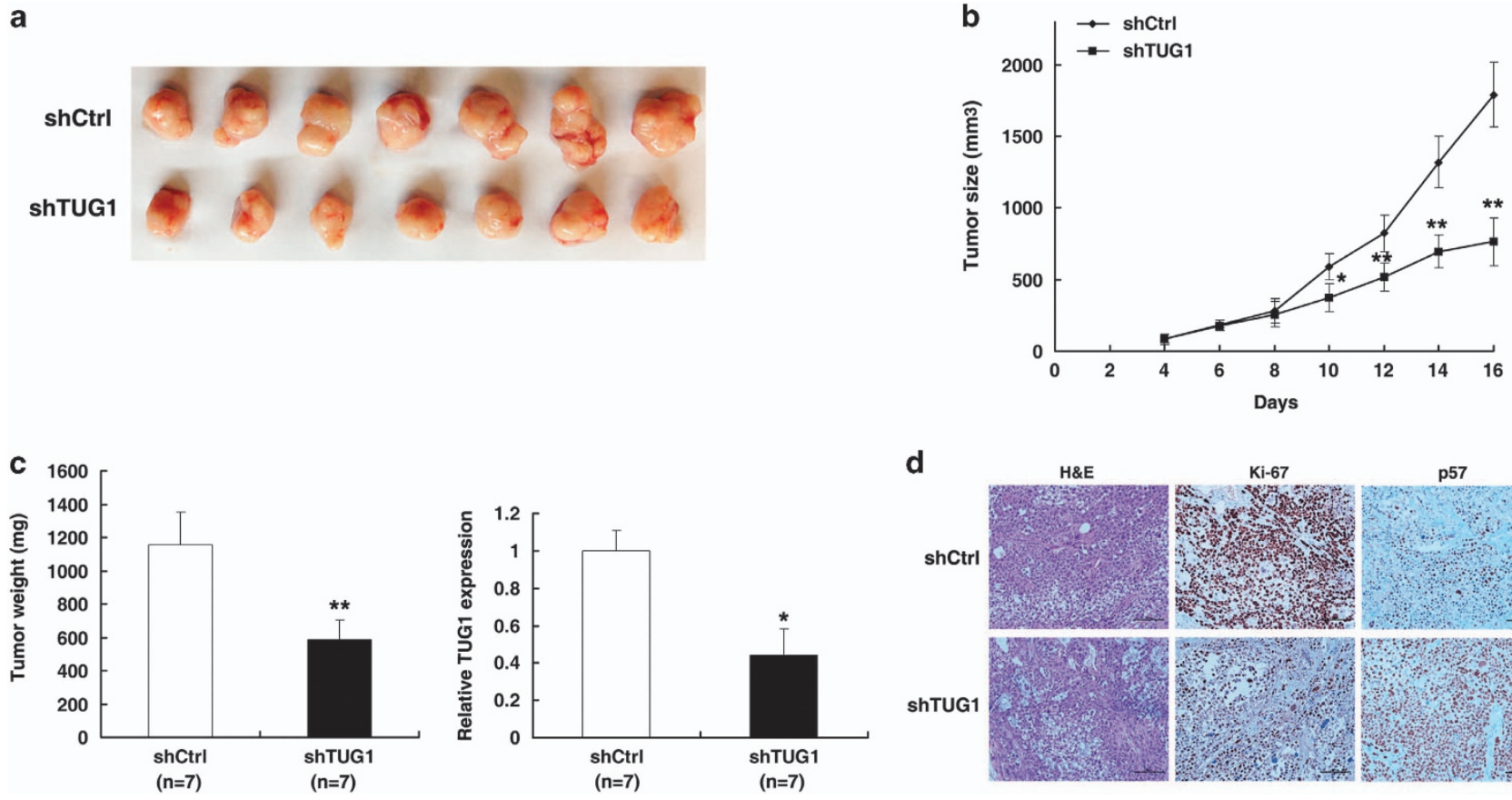

d

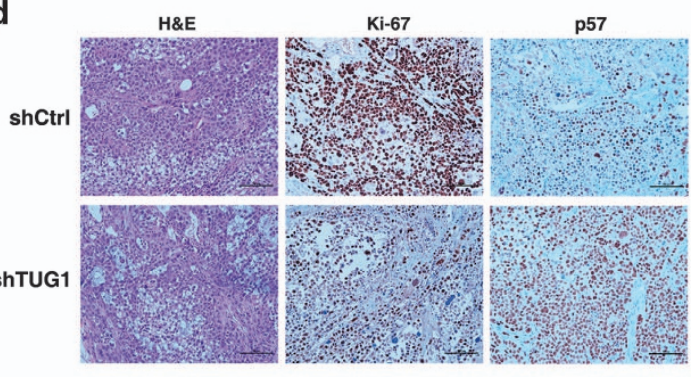

Figure 3 The impact of TUG1 on tumorigenesis in vivo. (a and $\mathbf{b})$ Scramble or shTUG1 was transfected into AGS cells, which were injected into nude mice ( $n=7$ ). The tumor volumes were calculated every 2 days after injection. The bars indicate S.D. (c) The tumor weights are shown as means of tumor weights \pm S.D. qRT-PCR was performed to detect the average expression of TUG1. (d) Histopathology of xenograft tumors. The tumor sections underwent H\&E staining and IHC staining using antibodies against Ki-67 and p57. Bar, $100 \mu \mathrm{m}$. Error bars indicate means \pm S.E.M. ${ }^{*} P<0.05,{ }^{* *} P<0.01$

cyclinD-CDK4/6 kinases or inactivation of the CKIs can result in cell cycle disorders and boost cell proliferation. ${ }^{37}$ The kinase activity of Cdk/cyclin complexes is tightly modulated by CKIs, which serve as brakes to halt cell cycle progression. ${ }^{38}$ In addition, CKIs act as tumor suppressors in various cancers, and aberrant methylation in the CKI gene promoter region has been linked to downregulation of gene expression, ${ }^{39}$ whereas PRC2-mediated histone methylation contributes to the repression of CKIs. ${ }^{40-44}$ Our results showed that the knockdown of TUG1 could obviously induce the expression of CKIs in an EZH2-dependent manner. Our results explained how CKIs are specifically regulated by PRC2, due in part to TUG1. Many IncRNAs modulate specific genetic loci through recruiting and binding to PRC2 protein complexes, and PRC2-mediated epigenetic regulation has a crucial role in the process of tumor development. ${ }^{13}$

The functional roles of p15, p16 and p21 have been illustrated in GC, ${ }^{28,29}$ and our previous research showed that $\mathrm{p} 27$ serves as a tumor suppressor in GC. ${ }^{30}$ However, the functional role of $p 57$ in $\mathrm{GC}$ remains unclear. Our results determined that $\mathrm{p} 57$ can serve as a tumor suppressor in GC. Many results have demonstrated that the cell cycle can be regulated by IncRNAs. ${ }^{45}$ These results showed that TUG1 could have a key role in the cell cycle of GC.

In summary, our study identified a TUG1-mediated regulator of the GC cell cycle and cell proliferation. TUG1 may enrich a mechanistic link between IncRNAs and the cell cycle regulation pathway, and TUG1, as a member of PRC2-mediated epigenetic regulation, participates in the occurrence and development of GC. This IncRNA may serve as a target for new therapies in GC.

\section{Materials and Methods}

Tissue collection and ethics statement. A total of 100 patients analyzed in this study underwent resection of the primary GC at the First Affiliated Hospital of Nanjing Medical University. The study was approved by the Research Ethics Committee of Nanjing Medical University (Nanjing, Jiangsu, China), and written informed consent was obtained from all patients. The clinicopathological characteristics of the GC patients are summarized in Table 1.

RNA extraction and qRT-PCR analyses. Total RNA was extracted from tissues or cultured cells using TRIzol reagent (Invitrogen, Carlsbad, CA, USA). For qRT-PCR, RNA was reverse transcribed to cDNA by using a Reverse Transcription Kit (Takara, Dalian, China). Real-time PCR analyses were performed with SYBR Green (Takara). The results were normalized to the expression of $\beta$-actin. The rest of the primers are listed in Supplementary Table 1.

Cell culture. Four GC cell lines (AGS, SGC-7901, BGC-823 and MGC-803) and a normal gastric epithelial cell line (GES-1) were purchased from the Institute of Biochemistry and Cell Biology of the Chinese Academy of Sciences (Shanghai, China). The cells were cultured in RPMI 1640 or DMEM (GIBCO-BRL) medium supplemented with $10 \%$ fetal bovine serum (10\% FBS), $100 \mathrm{U} / \mathrm{ml}$ penicillin and $100 \mathrm{mg} / \mathrm{ml}$ streptomycin in humidified air at $37^{\circ} \mathrm{C}$ with $5 \% \mathrm{CO}_{2}$.

Cell proliferation assays. Cell proliferation was monitored using the Cell Proliferation Reagent Kit I (MTT) (Roche, Basel, Switzerland). The transfected cells were plated in 96-well plates (3000 cells per well). Cell proliferation was determined every $24 \mathrm{~h}$ following the manufacturer's protocol. For the colony formation assay, a certain number of transfected cells were placed into each well of a six-well plate and maintained in media containing 10\% FBS for 2 weeks, replacing the medium every 4 days. Colonies were fixed with methanol and stained with $0.1 \%$ crystal violet (Sigma-Aldrich, St. Louis, MO, USA) in PBS for $15 \mathrm{~min}$. The colony formation was determined by counting the number of stained colonies. Triplicate wells were measured in each treatment group. 
a

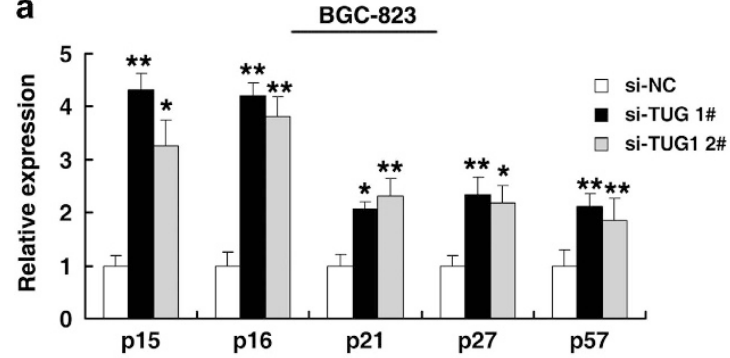

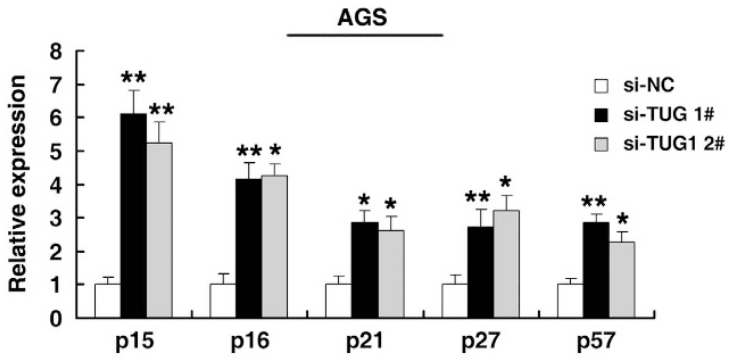
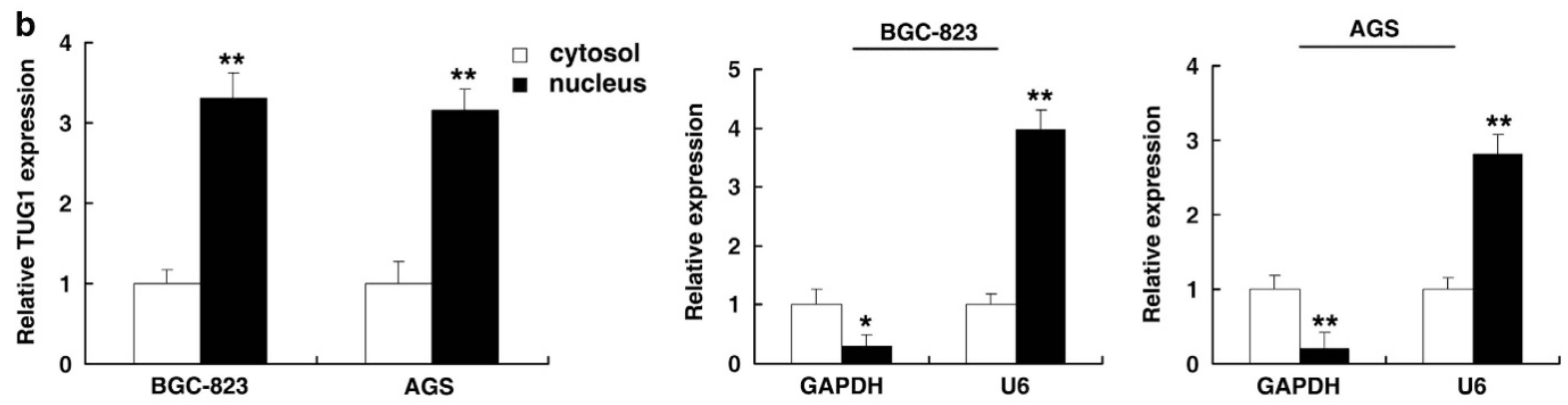

c

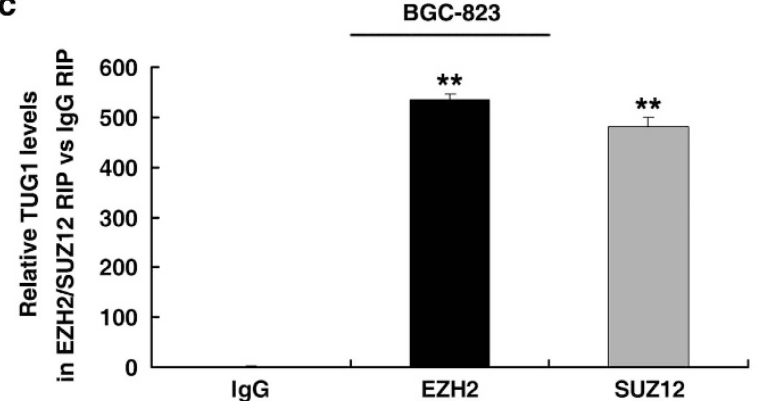

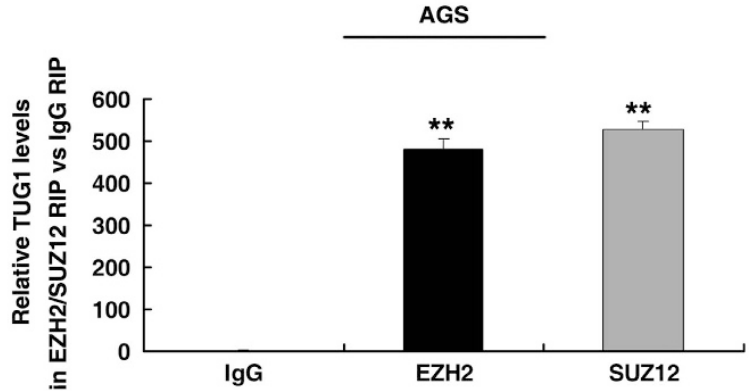

Figure 4 TUG1 is associated with PRC2 in GC. (a) The expression of p15, p16, p21, p27 and p57 was determined after knockdown of TUG1 using qRT-PCR. (b) TUG1 nuclear localization, as identified using qRT-PCR in fractionated BGC-823 and AGS cells. After nuclear and cytosolic separation, RNA expression levels were measured by qRTPCR. GAPDH was used as a cytosolic marker, and U6 was used as a nuclear marker. (c) RIP experiments were performed, and the coprecipitated RNA was subjected to qRTPCR for TUG1. The fold enrichment of TUG1 in RIPs is relative to its matching IgG control RIP. ${ }^{\star} P<0.05,{ }^{*} P<0.01$

Western blot assay and antibodies. Cell protein lysates were separated by $10 \%$ SDS-polyacrylamide gel electrophoresis, transferred to $0.22-\mu \mathrm{m} \mathrm{NC}$ membranes (Sigma-Aldrich) and incubated with specific antibodies. Autoradiograms were quantified by densitometry (Quantity One software; Bio-Rad, Hercules, CA, USA), and $\beta$-actin antibody was used as the control. Anti-p57 was purchased from Cell Signaling Technology, Inc (Boston, MA, USA). Antibodies against EZH2 and SUZ12 were purchased from Abcam.

Flow cytometric analysis. Transfected cells were harvested after transfection by trypsinization. Cells for cell cycle analysis were stained with propidium oxide using the CycleTEST PLUS DNA Reagent Kit (BD Biosciences, Franklin Lakes, NJ, USA) according to the protocol and analyzed by FACScan (BD Biosciences). The percentages of cells in G0-G1, S and G2-M phase were counted and compared.

Immunohistochemistry. Immunohistochemistry was performed as previously described. ${ }^{46}$ The anti-EZH2 antibody was purchased from Abcam.

Xenograft study. Five-week-old athymic BALB/c mice were maintained under specific pathogen-free conditions and manipulated according to protocols approved by the Shanghai Medical Experimental Animal Care Commission. AGS cells were transfected with Scramble or shTUG1. After $48 \mathrm{~h}$, the cells were collected and injected into either side of the posterior flank of the nude mouse. Tumor volumes were examined every 2 days when the implantations started to grow. Tumor volumes (length $\times$ width $^{2} \times 0.5$ ) and weights were measured every 2 days in mice from the control (seven mice) or shTUG1 (seven mice) groups. Sixteen days after injection, the mice were killed and the tumor weights were measured.

Transfection of GC cells. GC cells were transfected with siRNA oligonucleotides with plasmids using Lipofectamine 2000 (Invitrogen, USA) according to the manufacturer's protocol. The nucleotide sequences of siRNA for TUG1 were (siRNA 1\# (sense 5'-GCUUGGCUUCUAUUCUGAAUCCUUU-3', antisense 5'-AAAGGAUU CAGAAUAGAAGCCAAGC-3'); siRNA 2\# (sense 5'-CAGCUGUUACCAUUCAACU UCUUAA-3', antisense 5'-UUAAGAAGUUGAAUGGUAACAGCUG-3'); Negative control siRNA (si-NC) was purchased from Invitrogen (Invitrogen, USA). The p57 and p21 siRNAs were purchased from Santa Cruz (Dallas, TX, USA) (cat. no. sc-35751 and sc-29427). The shTUG1 was cloned into the pENTR ${ }^{\text {TM/U6 }}$ vector, as previously described. ${ }^{24}$ The sequence of $p 57$ was synthesized and subcloned into a pCDNA3.1 vector (Invitrogen, Shanghai, China). After transfection, the cells were harvested for further studies.

Subcellular fractionation location. The separation of the nuclear and cytosolic fractions was performed using the PARIS Kit (Life Technologies, Carlsbad, CA, USA) according to the manufacturer's instructions.

ChIP assays. ChIP assays were performed using the EZ-CHIP KIT according to the manufacturer's instructions (Millipore, Billerica, MA, USA). EZH2 and SUZ12 antibodies were obtained from Abcam. H3 trimethyl Lys 27 antibody was purchased from Millipore. The ChIP primer sequences are listed in 
Supplementary Table 3. Quantification of immunoprecipitated DNA was performed using QPCR with SYBR Green Mix (Takara). The ChIP data were calculated as a percentage relative to the input DNA using the equation $2^{[\text {[nput } \mathrm{Ct} \text { - Target } \mathrm{Ct}]}$ $\times 0.1 \times 100$.
RNA immunoprecipitation. RIP experiments were performed using a Magna RIP RNA-Binding Protein Immunoprecipitation Kit (Millipore) according to the manufacturer's instructions. Antibodies for RIP assays against EZH2 and SUZ12 were purchased from Abcam.
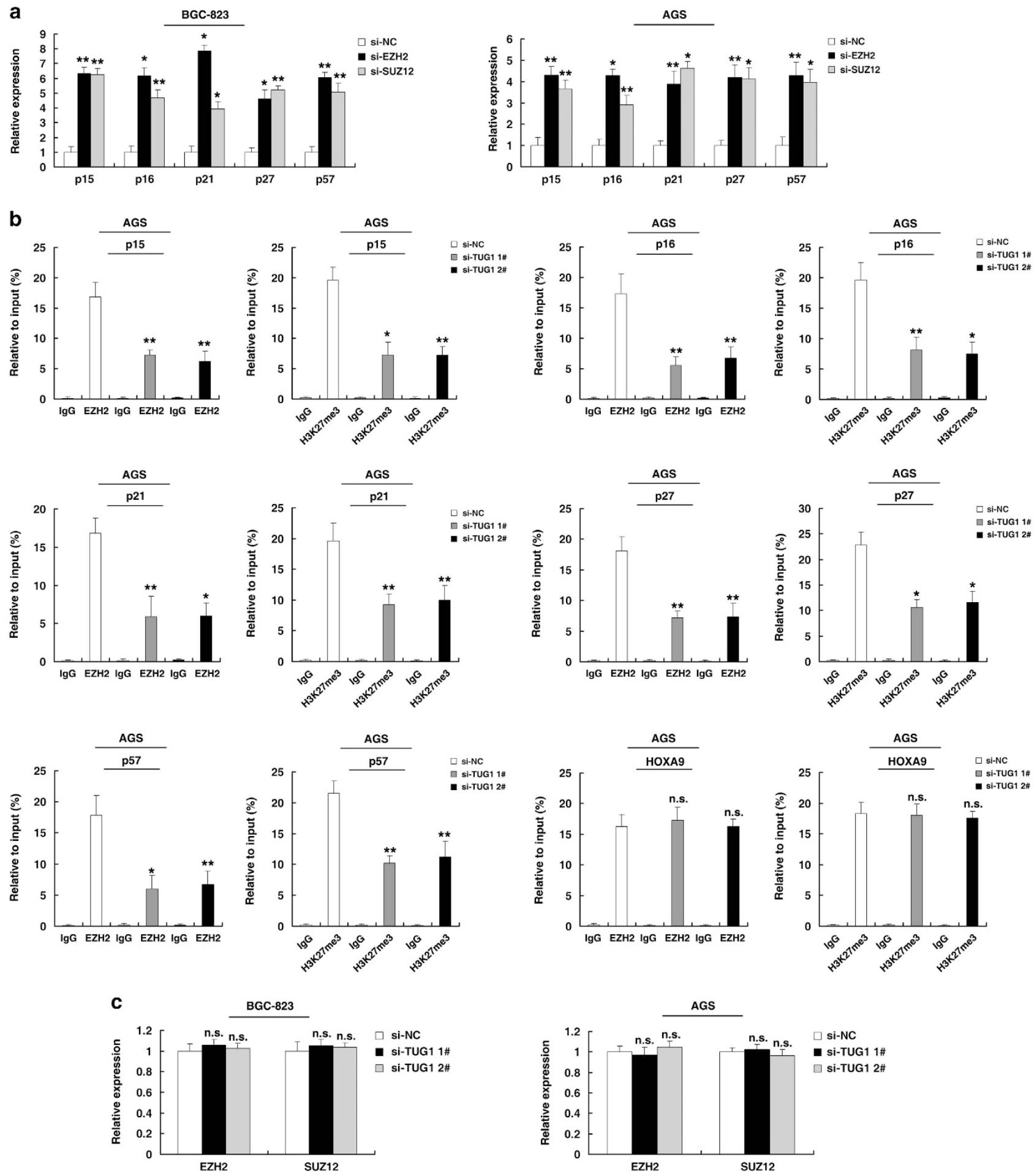

Figure 5 TUG1 is required to target PRC2 occupancy and activity to epigenetically regulate the expression of CKIs, thus regulating GC cell cycle and proliferation. (a) The expression of p15, p16, p21, p27 and p57 in BGC-823 and AGS cells, after knockdown of EZH2 and SUZ12. (b) ChIP-qPCR of H3K27me3 and EZH2 of the promoter region of the p15, p16, p21, p27 and p57 locus after siRNA treatment targeting si-NC or si-TUG1 in AGS cells. Antibody enrichment was quantified relative to the amount of input DNA. Antibody directed against IgG was used as a negative control. (c) The expression of EZH2 and SUZ12 in BGC-823 and AGS cells, after knockdown of TUG1. ${ }^{*} P<0.05$, ${ }^{\star *} P<0.01$ 


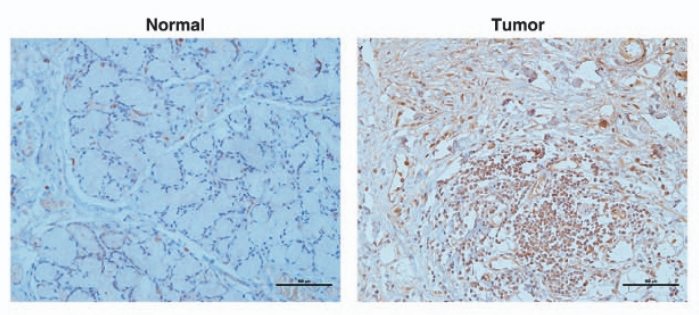

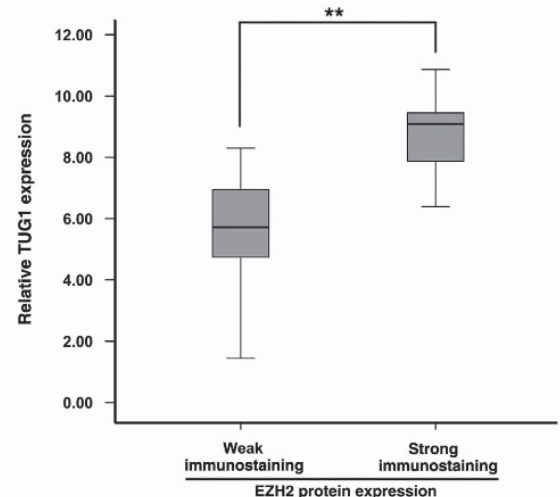

BGC-823

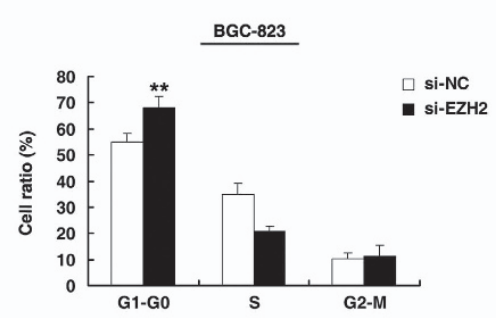

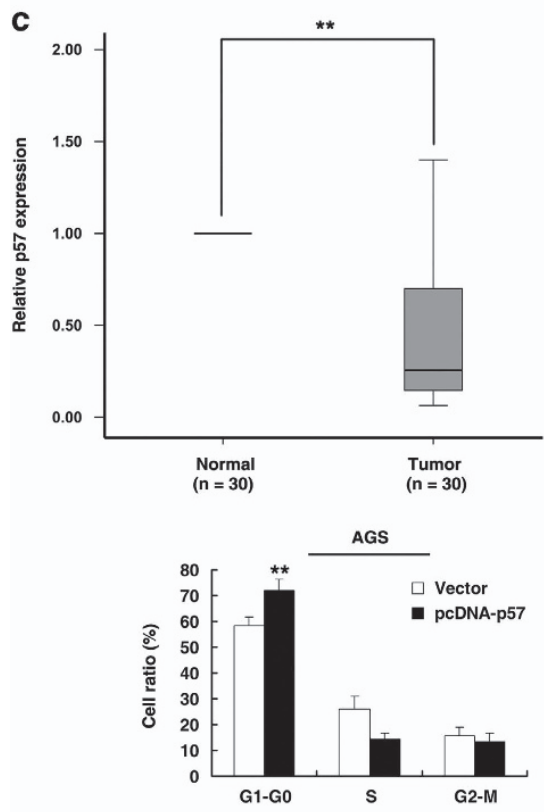
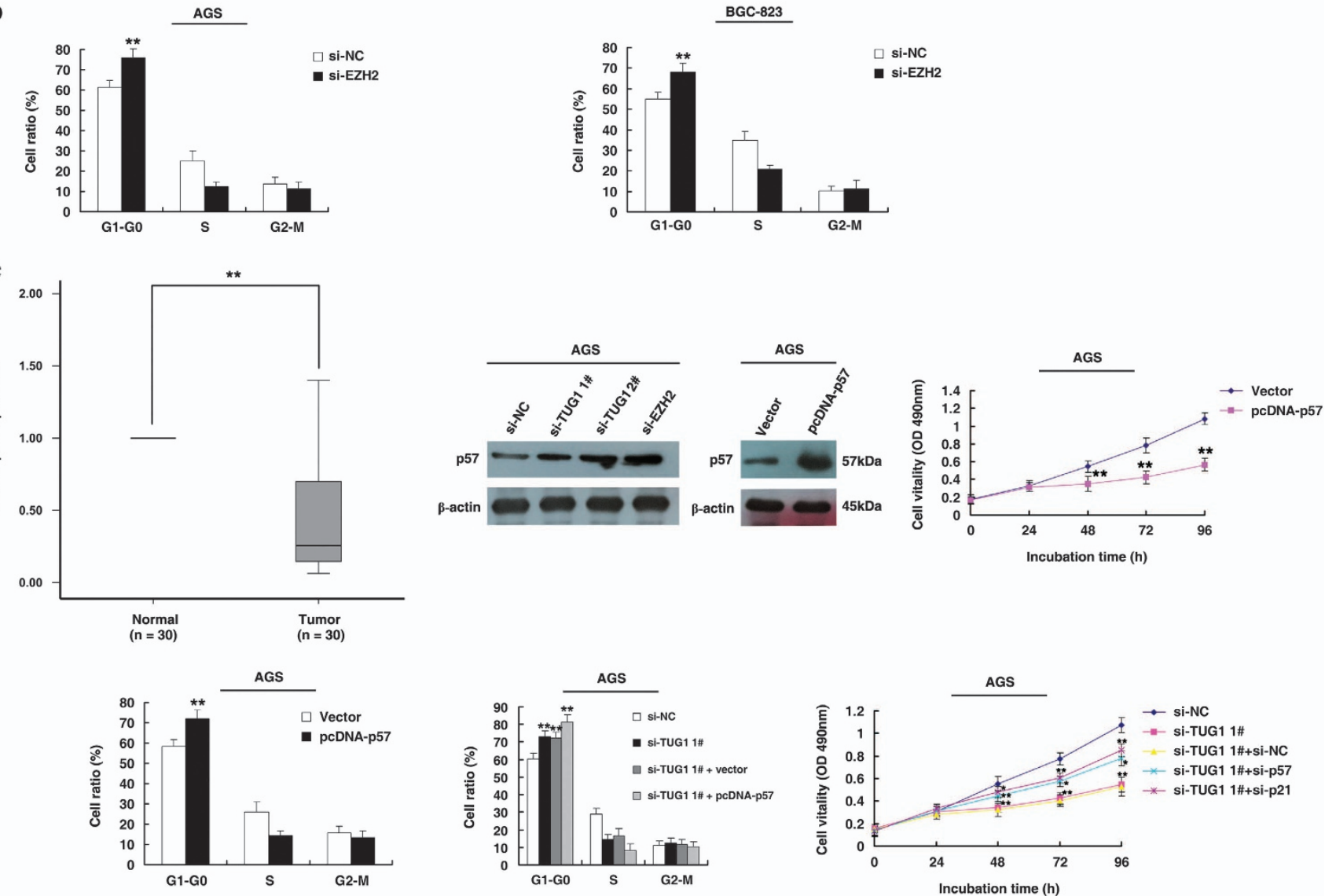

Figure 6 The role of p57 in GC. (a) Immunohistochemistry was used to detect the expression of EZH2 protein in 30 pairs of GC with corresponding non-tumor tissues. Bar, $100 \mu \mathrm{m}$. The immunoreactivity of EZH2 protein in GC tissues showed a statistically significant positive correlation with the relative level of TUG1 expression. (b) AGS and BGC-823 cells transfected with si-NC/si-EZH2. Forty-eight hours after transfection, the cells were analyzed using flow cytometry. (c) As determined by qRT-PCR assays, the level of p57 was downregulated in 30 pairs of GC tissues. Western blot assays detected the expression of p57 after transfection. AGS cells were transfected with Vector/p57. Fortyeight hours after transfection, the cells were analyzed using MTT assays and flow cytometry. AGS cells were transfected with si-NC/si-TUG1/si-TUG1+vector and transfected with si-TUG1 followed by transfection with pcDNA-p57. After transfection, the cells were stained and analyzed using flow cytometry. MTT analysis of cell proliferation by co-transfection (si-NC, si-TUG1 1\#, si-TUG1 1\#+si-NC, si-TUG1 1\#+si-p57, si-TUG1 1\#+si-p21). ${ }^{*} P<0.05,{ }^{* *} P<0.01$

Statistical analysis. All statistical analyses were performed using SPSS 20.0 software (IBM, SPSS, Armonk, NY, USA). The significant differences between groups were estimated by Student's $t$-test or $\chi 2$ test, as appropriate. The OS rates were calculated using the Kaplan-Meier method, with the log-rank test applied for comparison. The survival data were evaluated using univariate and multivariate Cox proportional hazards models. Variables with a value of $P<0.05$ in the univariate analysis were used in subsequent multivariate analyses on the basis of Cox regression analyses. Two-sided $P$-values were calculated, and a probability level of 0.05 was chosen for statistical significance.

\section{Conflict of Interest}

The authors declare no conflict of interest.

Acknowledgements. The work was supported by the National Natural Science Foundation of China (grant no. 81502071 and 81401873 ). This study is founded by the Natural Science Foundation of China (81372321 and 81572261 to Lin Xu; 81472200 to Rong Yin), Jiangsu Provincial Special Program of Medical Science (BL2012030 to Lin Xu). 
1. Coupland VH, Lagergren J, Luchtenborg M, Jack RH, Allum W, Holmberg L et al. Hospital volume, proportion resected and mortality from oesophageal and gastric cancer: a population-based study in England, 2004-2008. Gut 2013; 62: 961-966.

2. Camargo MC, Kim WH, Chiaravalli AM, Kim KM, Corvalan AH, Matsuo K et al. Improved survival of gastric cancer with tumour Epstein-Barr virus positivity: an international pooled analysis. Gut 2014; 63: 236-243.

3. Amaral PP, Dinger ME, Mercer TR, Mattick JS. The eukaryotic genome as an RNA machine. Science 2008; 319: 1787-1789.

4. Guttman M, Amit I, Garber M, French C, Lin MF, Feldser D et al. Chromatin signature reveals over a thousand highly conserved large non-coding RNAs in mammals. Nature 2009; 458 : 223-227.

5. Nagano T, Fraser P. No-nonsense functions for long noncoding RNAs. Cell 2011; 145: 178-181.

6. Amaral PP, Mattick JS, Noncoding RNA. in development. Mamm Genome 2008; 19 : 454-492.

7. Blackshaw S, Harpavat S, Trimarchi J, Cai L, Huang H, Kuo WP et al. Genomic analysis of mouse retinal development. PLOS Biol 2004; 2: E247.

8. Dinger ME, Amaral PP, Mercer TR, Pang KC, Bruce SJ, Gardiner BB et al. Long noncoding RNAs in mouse embryonic stem cell pluripotency and differentiation. Genome Res 2008; 18 : 1433-1445.

9. Rinn JL, Kertesz M, Wang JK, Squazzo SL, Xu X, Brugmann SA et al. Functional demarcation of active and silent chromatin domains in human HOX loci by noncoding RNAs. Cell 2007; 129: 1311-1323.

10. Ginger MR, Shore AN, Contreras A, Rijnkels M, Miller J, Gonzalez-Rimbau MF et al. A noncoding RNA is a potential marker of cell fate during mammary gland development. Proc Natl Acad Sci USA 2006; 103: 5781-5786.

11. Cesana M, Cacchiarelli D, Legnini I, Santini T, Sthandier O, Chinappi $M$ et al. A long noncoding RNA controls muscle differentiation by functioning as a competing endogenous RNA. Cell 2011; 147: 358-369.

12. Ishii N, Ozaki K, Sato H, Mizuno H, Saito S, Takahashi A et al. Identification of a novel noncoding RNA, MIAT, that confers risk of myocardial infarction. J Hum Genet 2006; 51: 1087-1099.

13. Gupta RA, Shah N, Wang KC, Kim J, Horlings HM, Wong DJ et al. Long non-coding RNA HOTAIR reprograms chromatin state to promote cancer metastasis. Nature 2010; 464 : 1071-1076.

14. Yuan SX, Yang F, Yang Y, Tao QF, Zhang J, Huang G et al. Long noncoding RNA associated with microvascular invasion in hepatocellular carcinoma promotes angiogenesis and serves as a predictor for hepatocellular carcinoma patients' poor recurrence-free survival after hepatectomy. Hepatology 2012; 56: 2231-2241.

15. Khaitan D, Dinger ME, Mazar J, Crawford J, Smith MA, Mattick JS et al. The melanomaupregulated long noncoding RNA SPRY4-IT1 modulates apoptosis and invasion. Cancer Res 2011; 71: 3852-3862.

16. Zhang EB, Kong R, Yin DD, You LH, Sun M, Han L et al. Long noncoding RNA ANRIL indicates a poor prognosis of gastric cancer and promotes tumor growth by epigenetically silencing of miR-99a/miR-449a. Oncotarget 2014; 5: 2276-2292.

17. Poliseno L, Salmena L, Zhang J, Carver B, Haveman WJ, Pandolfi PP. A codingindependent function of gene and pseudogene mRNAs regulates tumour biology. Nature 2010; 465: 1033-1038.

18. Liu XH, Sun M, Nie FQ, Ge YB, Zhang EB, Yin DD et al. Lnc RNA HOTAIR functions as a competing endogenous RNA to regulate HER2 expression by sponging miR-331-3p in gastric cancer. Mol Cancer 2014; 13: 92.

19. Khalil AM, Guttman M, Huarte M, Garber M, Raj A, Rivea Morales D et al. Many human large intergenic noncoding RNAs associate with chromatin-modifying complexes and affect gene expression. Proc Natl Acad Sci USA 2009; 106: 11667-11672.

20. Young TL, Matsuda T, Cepko CL. The noncoding RNA taurine upregulated gene 1 is required for differentiation of the murine retina. Curr Biol 2005; 15: 501-512.

21. Han Y, Liu Y, Gui Y, Cai Z. Long intergenic non-coding RNA TUG1 is overexpressed in urothelial carcinoma of the bladder. J Surg Oncol 2013; 107: 555-559.

22. Zhang $Q$, Geng PL, Yin P, Wang XL, Jia JP, Yao J. Down-regulation of long non-coding RNA TUG1 inhibits osteosarcoma cell proliferation and promotes apoptosis. Asian Pac J Cancer Prev 2013; 14: 2311-2315

23. Xu Y, Wang J, Qiu M, Xu L, Li M, Jiang F et al. Upregulation of the long noncoding RNA TUG1 promotes proliferation and migration of esophageal squamous cell carcinoma. Tumour Biol 2014; 36: 1643-1651.

24. Zhang EB, Yin DD, Sun M, Kong R, Liu XH, You LH et al. P53-regulated long non-coding RNA TUG1 affects cell proliferation in human non-small cell lung cancer, partly through epigenetically regulating HOXB7 expression. Cell Death Dis 2014; 5: e1243.

25. Prensner JR, lyer MK, Balbin OA, Dhanasekaran SM, Cao Q, Brenner JC et al. Transcriptome sequencing across a prostate cancer cohort identifies PCAT-1, an unannotated lincRNA implicated in disease progression. Nat Biotechnol 2011; 29: 742-749.
26. Kotake Y, Nakagawa T, Kitagawa K, Suzuki S, Liu N, Kitagawa M et al. Long non-coding RNA ANRIL is required for the PRC2 recruitment to and silencing of p15(INK4B) tumor suppressor gene. Oncogene 2011; 30: 1956-1962.

27. Cao R, Zhang Y. SUZ12 is required for both the histone methyltransferase activity and the silencing function of the EED-EZH2 complex. Mol Cell 2004; 15: 57-67.

28. Matsumoto Y, Marusawa H, Kinoshita K, Niwa Y, Sakai Y, Chiba T. Up-regulation of activation-induced cytidine deaminase causes genetic aberrations at the CDKN2b-CDKN2a in gastric cancer. Gastroenterology 2010; 139: 1984-1994.

29. Kang ZH, Wang CY, Zhang WL, Zhang JT, Yuan $\mathrm{CH}$, Zhao PW et al. Histone deacetylase HDAC4 promotes gastric cancer SGC-7901 cells progression via p21 repression. PLoS One 2014; 9: e98894.

30. Sun M, Liu XH, Li JH, Yang JS, Zhang EB, Yin DD et al. MiR-196a is upregulated in gastric cancer and promotes cell proliferation by downregulating p27(kip1). Mol Cancer Ther 2012; 11: $842-852$.

31. Derrien T, Johnson R, Bussotti G, Tanzer A, Djebali S, Tilgner H et al. The GENCODE v7 catalog of human long noncoding RNAs: analysis of their gene structure, evolution, and expression. Genome Res 2012; 22: 1775-1789.

32. Cabili MN, Trapnell C, Goff L, Koziol M, Tazon-Vega B, Regev A et al. Integrative annotation of human large intergenic noncoding RNAs reveals global properties and specific subclasses. Genes Dev 2011; 25: 1915-1927.

33. Cao WJ, Wu HL, He BS, Zhang YS, Zhang ZY. Analysis of long non-coding RNA expression profiles in gastric cancer. World J Gastroenterol 2013; 19: 3658-3664.

34. Gutschner T, Hammerle M, Eissmann M, Hsu J, Kim Y, Hung G et al. The noncoding RNA MALAT1 is a critical regulator of the metastasis phenotype of lung cancer cells. Cancer Res 2013; 73: 1180-1189.

35. Hu Y, Wang J, Qian J, Kong X, Tang J, Wang Y et al. Long noncoding RNA GAPLINC regulates CD44-dependent cell invasiveness and associates with poor prognosis of gastric cancer. Cancer Res 2014; 74: 6890-6902.

36. Xu TP, Liu XX, Xia R, Yin L, Kong R, Chen WM et al. SP1-induced upregulation of the long noncoding RNA TINCR regulates cell proliferation and apoptosis by affecting KLF2 mRNA stability in gastric cancer. Oncogene 2015; 34: 5648-5661.

37. Sherr CJ, McCormick F. The RB and p53 pathways in cancer. Cancer Cell 2002; 2: 103-112.

38. Lim S, Kaldis P. Cdks, cyclins and CKIs: roles beyond cell cycle regulation. Development 2013; 140: 3079-3093.

39. Daa T, Kashima K, Kondo Y, Yada N, Suzuki M, Yokoyama S. Aberrant methylation in promoter regions of cyclin-dependent kinase inhibitor genes in adenoid cystic carcinoma of the salivary gland. APMIS 2008; 116: 21-26.

40. Paul TA, Bies J, Small D, Wolff L. Signatures of polycomb repression and reduced H3K4 trimethylation are associated with p15INK4b DNA methylation in AML. Blood 2010; 115: 3098-3108.

41. Aoki R, Chiba T, Miyagi S, Negishi M, Konuma T, Taniguchi $\mathrm{H}$ et al. The polycomb group gene product Ezh2 regulates proliferation and differentiation of murine hepatic stem progenitor cells. J Hepatol 2010; 52: 854-863.

42. Chen H, Gu X, Su IH, Bottino R, Contreras JL, Tarakhovsky A et al. Polycomb protein Ezh2 regulates pancreatic beta-cell Ink4a/Arf expression and regeneration in diabetes mellitus. Genes Dev 2009; 23: 975-985.

43. Fan T, Jiang S, Chung N, Alikhan A, Ni C, Lee CC et al. EZH2-dependent suppression of a cellular senescence phenotype in melanoma cells by inhibition of p21/CDKN1A expression. Mol Cancer Res 2011; 9: 418-429.

44. Yang X, Karuturi RK, Sun F, Aau M, Yu K, Shao R et al. CDKN1C (p57) is a direct target of $\mathrm{EZH} 2$ and suppressed by multiple epigenetic mechanisms in breast cancer cells. PLoS One 2009; 4: e5011.

45. Kitagawa M, Kitagawa K, Kotake $\mathrm{Y}$, Niida $\mathrm{H}$, Ohhata T. Cell cycle regulation by long noncoding RNAs. Cell Mol Life Sci 2013; 70: 4785-4794.

46. Liao WT, Wang X, Xu LH, Kong QL, Yu CP, Li MZ et al. Centromere protein $\mathrm{H}$ is a novel prognostic marker for human nonsmall cell lung cancer progression and overall patient survival. Cancer 2009; 115: 1507-1517.

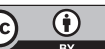

Cell Death and Disease is an open-access journal published by Nature Publishing Group. This work is licensed under a Creative Commons Attribution 4.0 International License. The images or other third party material in this article are included in the article's Creative Commons license, unless indicated otherwise in the credit line; if the material is not included under the Creative Commons license, users will need to obtain permission from the license holder to reproduce the material. To view a copy of this license, visit http://creativecommons.org/licenses/by/4.0/ 